\title{
Avances a los diez años de vigencia de la Ley del Procedimiento Administrativo General
}

\section{Ten years in force of the General Administrative Procedure Law: progress}

MILAGROS MARAVÍ SUMAR*

Resumen: En el artículo, la autora analiza lo ocurrido en estos diez años con respecto a temas como los principios del procedimiento administrativo, la simplificación administrativa, los procedimientos sancionadores, el control difuso en sede administrativa y el silencio administrativo.

Palabras clave: Ley del Procedimiento Administrativo General - principios del procedimiento administrativo - simplificación administrativa - procedimiento sancionador - control difuso en sede administrativa - silencio administrativo

Abstract: In this article the author analyzes what happened in these ten years regarding issues such as administrative procedure principles, administrative simplification, sanctioning proceedings, administrative site diffuse control and administrative silence.

Key words: General Administrative Procedure Law - principles of the administrative procedure - administrative simplification - sanctioning proceeding diffuse control in administrative site - administrative silence

CONTENIDO: INTRODUCCIÓN.- I. PRINCIPIOS DEL PROCEDIMIENTO ADMINISTRATIVO.- II. SIMPLIFICACIÓN ADMINISTRATIVA.- III. LOS PROCEDIMIENTOS SANCIONADORES.- IV. EL CONTROL DIFUSO DE LA ADMINISTRACIÓN.- V. EL SILENCIO ADMINISTRATIVO.- VI. CONCLUSIÓN.

\section{INTRODUCCIÓN}

Se cumplen diez años de la vigencia de la Ley del Procedimiento Administrativo General 27444 (LPAG) y, habida cuenta del tiempo transcurrido y de la experiencia desarrollada, es preciso destacar que el balance es positivo en términos de técnica legislativa, lo cual ha sido objeto de reflexión nacional y comparada en los congresos nacionales de Derecho

Es abogada graduada por la Pontificia Universidad Católica del Perú y cuenta con título de maestría en Administración pública por el Instituto Ortega y Gasset de la Universidad Complutense de Madrid. Como socia del Estudio Rubio, Leguía, Normand \& Asociados lidera el área de Derecho administrativo. Es profesora de la maestría de Derecho Administrativo Económico de la Universidad Continental y del diplomado de Derecho Administrativo Económico de la UPC en Lima. Correo electrónico: mmaravi@ rubio.pe. 
administrativo organizados por la Pontificia Universidad Católica del Perú y por nuestra Asociación Peruana de Derecho Administrativo, las cuales han acompañado su vigencia y han sido recogidos en sendas publicaciones ${ }^{1}$. Nuestra ley es una buena norma que ha regido el desarrollo de los procedimientos administrativos en los ámbitos nacional, regional y municipal.

Más allá del aspecto técnico-jurídico, el enfoque y propósito de la LPAG ha sido lo fundamental para los avances en su aplicación. Como señala Juan Carlos Morón:

El nuevo Derecho Administrativo que se propugna, como forma superada del Derecho Administrativo permiso, ha adoptado asumir como suyo el conjunto de valores constitucionales de respeto a la persona humana a sus derechos e intereses, en balance con el ejercicio de las potestades públicas. El artículo III del Título Preliminar de la Ley es clave para entender esta orientación:

«La presente Ley tiene por finalidad establecer el régimen jurídico aplicable para que la actuación de la Administración Pública sirva a la protección del interés general, garantizando los derechos e intereses de los administrados y con sujeción al ordenamiento constitucional y jurídico en general.

En tal sentido, algunas de las líneas matrices de la nueva ley, en aras de proyectarse como una base para el nuevo Derecho Administrativo democrático, son: delimitar la discrecionalidad administrativa, consagrar derechos sustantivos y previsibilidad a los actos de la Administración, incorporar reglas de modalidad administrativa, darle garantías a los administrados que las entidades respondan los perjuicios ocasionados, erradicar la cultura del secreto en la Administración, promover la eficiencia de las decisiones administrativas, propiciar el debido procedimiento previo a los administrados antes de la tutela judicial efectiva, entre otros» ${ }^{2}$.

Este objetivo fue clave desde un inicio y ya lo decía el año 2001 el mismo Jorge Danós, presidente de la comisión que elaboró la LPAG — la cual, dicho sea de paso, tuve el honor de integrar-:

En nuestra opinión, el impacto de esta nueva ley reguladora de las relaciones entre la Administración Pública y los ciudadanos deberá evaluarse tomando en cuenta que modernizará una normativa que

1 Derecho administrativo contemporáneo. Ponencias del Segundo Congreso Nacional de Derecho Administrativo. Lima: Palestra Editores, 2007; El Derecho administrativo y la modernización del Estado peruano. Ponencias del Tercer Congreso Nacional de Derecho Administrativo. Lima: Grijley, 2008; Modernizando el Estado para un país mejor. Ponencias del Cuarto Congreso Nacional de Derecho Administrativo. Lima: Palestra Editores, 2010.

2 MoRón, Juan Carlos. «Comentarios a la Ley de procedimiento administrativo general». Gaceta Jurídica. Octava edición. Lima 2009, p. 15. 
data desde hace más de 30 años. La citada ley regula el procedimiento administrativo fiel a los principios democráticos propios de un estado de derecho, con una opción clara a favor de las garantías de los ciudadanos, orientación ésta, que requerirá ser internalizada por las prácticas anquilosadas, y asumida también por los propios ciudadanos para que su eficacia sea plena. En tal sentido, es responsabilidad de la doctrina de Derecho Administrativo nacional formular los desarrollos dogmáticos para el análisis y difusión de los alcances de la nueva ley, evitando la práctica lamentablemente tan extendida en las publicaciones nacionales de limitarse al simple comentario exegético de un texto legal, omitiendo la explicación del fundamento y la razón de ser de las instituciones elaboradas por el Derecho Administrativo que son indispensables para la correcta comprensión de una norma por parte de los operadores administrativos o privados ${ }^{3}$.

En ese sentido, en estos diez años de vigencia de la LPAG se ha desarrollado abundante doctrina nacional que apunta a sustentar su aplicación en beneficio de la ciudadanía, con vigencia de sus derechos y, particularmente, del debido proceso, así como también de lo referido a las entidades públicas, a fin de orientar y potenciar la eficiencia y eficacia de la administración pública.

Siempre una ley resulta perfectible, y eso se ha hecho evidente, por ejemplo, al modificarse las normas de notificaciones para generalizar la notificación por correo electrónico como medio de primera prelación ${ }^{4}, \mathrm{o}$ al reemplazar el Diario Oficial El Peruano la farragosa publicación -en el papel — del Texto Único de Procedimientos Administrativos (TUPA) por su difusión en las páginas web institucionales y el portal del Estado5. En lo que se refiere al silencio administrativo, en cambio, se aprobó la Ley 29060 (Ley del Silencio Administrativo (LSA) y el decreto legislativo 1029 , que, si bien han impulsado la simplificación administrativa, han errado en técnica legislativa al extraer parte del tratamiento del silencio administrativo de la LPAG. Se trata de un error a enmendar y que pasa por (i) devolver el tratamiento del silencio administrativo a la LPAG; (ii) mantener algunas disposiciones de la LSA, pero en el contexto de la LPAG; (iii) derogar la LSA; y (iv) aclarar en la LPAG los supuestos de

3 DANós, Jorge. «El proceso de elaboración y aprobación de la nueva Ley del Procedimiento Administrativo General». En Comentarios a la Ley del Procedimiento Administrativo General, Ley $N^{\circ}$ 27444. Lima: ARA Editores, 2001, pp. 32-33.

4 «20.4. El administrado interesado o afectado por el acto que hubiera consignado en su escrito alguna dirección electrónica que conste en el expediente podrá ser notificado a través de ese medio siempre que haya dado su autorización expresa para ello. Para este caso no es de aplicación el orden de prelación dispuesto en el numeral 20.1». $\left(^{\star}\right)$ Numeral incluido por el artículo 1 del decreto legislativo 1029, publicado el 24 de junio de 2008.

5 Párrafo modificado por el artículo 1 de la ley 29091, publicada el 26 septiembre 2007: «38.3. El TUPA es publicado en el Portal de Servicios al Ciudadano y Empresas - PSCE, y en el Portal Institucional».

AVANCES A

LOS DIEZ AÑOS

DE VIGENCIA

DE LA LEY DEL

PROCEDIMIENTO

ADMINISTRATIVO

GENERAL

TEN YEARS

IN FORCE OF

THE GENERAL

ADMINISTRATIVE

PROCEDURE LAW:

PROGRESS 
aprobación automática y de silencio administrativo positivo y negativo. Más adelante trataremos este tema con mayor extensión.

\section{PRINCIPIOS DEL PROCEDIMIENTO ADMINISTRATIVO}

Uno de los avances más importantes de la LPAG ha sido la aplicación real de una serie de principios que guían la actuación de las entidades públicas y que buscan que los procedimientos administrativos o trámites que la ciudadanía en general y las empresas tienen que realizar sean lo más sencillos y simples posibles, y que se respete el debido proceso $-\mathrm{O}$ debido procedimiento administrativo- . Particularmente, este aspecto ha sido relevante.

El desarrollo práctico del principio del debido procedimiento administrativo se ha dado a partir de su reconocimiento en la LPAG. En su artículo 1.2 de su título preliminar, se establece lo siguiente:

Los administrados gozan de todos los derechos y garantías inherentes al debido procedimiento administrativo, que comprende el derecho a exponer sus argumentos, a ofrecer y producir pruebas y a obtener una decisión motivada y fundada en derecho. La institución del debido procedimiento administrativo se rige por los principios del Derecho Administrativo. La regulación propia del Derecho Procesal Civil es aplicable sólo en cuanto sea compatible con el régimen administrativo.

Por su parte, el desarrollo doctrinario y jurisprudencial ha consolidado su exigencia y vigencia. En ese sentido, la doctrina especializada establece que:

[...] la dimensión más conocida del derecho al debido proceso comprende una serie de derechos que forman parte de un estándar mínimo de garantía para los administrados, que a grandes rasgos, mutatis mutandi implican la aplicación a la sede administrativa de los derechos concebidos originariamente en la sede de los procedimientos jurisdiccionales. Por lo general, se suelen desprender, los siguientes subprincipios esenciales: el contradictorio, el derecho de defensa, el derecho a ser notificado, el acceso al expediente, el derecho de audiencia, el derecho a probar, entre otros $[\ldots]^{6}$.

Al respecto, la sentencia del Tribunal Constitucional de expediente 01387-2009-PA/TC, Lima, CONSORCIO DHMONT \& CG \& M S.A.C. señala lo siguiente:

El derecho al debido proceso administrativo puede ser entendido, en sentido positivo, como la regulación jurídica que de manera previa de-

6 Morón, Juan Carlos. «Comentarios a la nueva Ley del Procedimiento Administrativo General». Gaceta Jurídica. Octava edición. Lima, 2009, p. 64. 
limita la actuación de los órganos que conforman la Administración Pública para que ésta sea correcta y establece las garantías mínimas de protección a los derechos de los administrados, de modo que ninguna de las actuaciones de los órganos que conforman la Administración Pública dependa de su propio arbitrio, sino que se encuentran sujetas siempre a los procedimientos previamente establecidos en la ley.

Por otro lado, la sentencia del Tribunal Constitucional de expediente 003-2004-AI/T, LIMA, Mateo Eugenio Quispe —en representación del 1\% de los ciudadanos del distrito de Ancón- establece lo siguiente:

Es doctrina consolidada de este Colegiado que el derecho reconocido en el inciso 3) del artículo $139^{\circ}$ de la Constitución no sólo tiene una dimensión, por así decirlo, «judicial», sino que se extiende también a sede «administrativa». En efecto, el debido proceso está concebido como el cumplimiento de todas las garantías, requisitos y normas de orden público que deben observarse en las instancias procesales de todos los procedimientos, incluidos los administrativos, a fin de que las personas estén en condiciones de defender adecuadamente sus derechos ante cualquier acto del Estado que pueda afectarlos.

El derecho al debido proceso comprende, a su vez, un haz de derechos que forman parte de su estándar mínimo: al juez natural — jurisdicción predeterminada por la ley—, de defensa, a la pluralidad de instancias, a los medios de prueba y a un proceso sin dilaciones. En el caso de autos, la lesión al debido proceso se constata por el uso de un mecanismo inapropiado e indebido para la sanción de nulidad de contratos, más precisamente mediante una ordenanza.

Por lo anterior, se puede verificar que, a los diez años de vigencia de la LPAG, ha habido un avance en la posición de la población ante las entidades públicas por el desarrollo legislativo, doctrinario y jurisprudencial del debido procedimiento administrativo.

\section{SIMPLIFICACIÓN ADMINISTRATIVA}

Sin embargo, como es obvio y aun pasados diez años, en muchas entidades públicas la LPAG no se cumple. Es cierto que existen muchos casos de buenas prácticas ${ }^{7}$, como también de mejoras en los procedimientos y servicios puntuales de entidades en todo el país ${ }^{8}$, pero

7 Como las veinte sistematizadas por la Presidencia del Consejo de Ministros entre las que se encuentran las experiencias del Servicio de Administración Tributaria (SAT), de la Municipalidad de San Borja, de la Superintendencia Nacional de los Registros Públicos (SUNARP), del Organismo Supervisor de la Inversión en Energía y Mineria (OSINERGMIN) y de la Superintendencia Nacional de Administración Tributaria (SUNAT), o las que cada año premia "Ciudadanos al Día».

8 La Presidencia del Consejo de Ministros publica un consolidado de mejoras cada año. Para el 2010, la resolución ministerial 152-2011-PCM aprueba el «Consolidado de mejoras en los procedimientos administrativos y servicios prestados en exclusividad efectuadas por los entidades públicas de todos los niveles de gobierno durante todo el año 2010" en concordancia con la Política Nacional de

AVANCES A

LOS DIEZ AÑOS

DE VIGENCIA

DE LA LEY DEL

PROCEDIMIENTO ADMINISTRATIVO GENERAL

TEN YEARS

IN FORCE OF

THE GENERAL

ADMINISTRATIVE PROCEDURE LAW: PROGRESS 
eso no es suficiente porque, en la generalidad de casos, la ciudadanía no percibe que en las entidades públicas no la atienden privilegiando su satisfacción y el cumplimiento de las leyes, sino privilegiando las formalidades y solicitando requisitos excesivos que las entidades bien podrían obviar si compartieran información entre ellas, de manera que no habría razón para cobrar de más ni para demorarse en resolver.

La solución a este problema no es propiamente normativa de la LPAG. La solución es legal en el sentido de que se trata de una actividad de las entidades públicas y servidores públicos, los cuales se rigen bajo el principio de legalidad $-\mathrm{y}$ no bajo su libre albedrío-, lo que hace que sus obligaciones estén normadas. Sin embargo, como parece obvio - pero a veces no se asume como tal-, la aprobación de normas no cambia la realidad. En ese sentido, postulamos que la mejora en los procedimientos administrativos se producirá cuando parte de la exigencia a la gestión pública sea la optimización de la atención al ciudadano y la simplificación de procedimientos administrativos.

En ese sentido, para la aplicación de la LPAG en la parte referida al procedimiento administrativo — nos referimos al marco de la Ley Orgánica del Poder Ejecutivo — la presidencia del consejo de ministros, como órgano rector, ha desarrollado el subsistema de simplificación administrativa como parte del sistema de la gestión pública.

El artículo 46 de la Ley Orgánica del Poder Ejecutivo 29158 (LOPE) reconoce que entre los sistemas administrativos figura el de modernización de la gestión pública, del cual el Poder Ejecutivo es el órgano rector y, como tal, responsable de reglamentar y operar los sistemas administrativos que son aplicables a todas las entidades de la administración pública, independientemente de su nivel de gobierno.

La simplificación administrativa es un subsistema de la modernización de la gestión pública, el cual es competencia de la Presidencia del Consejo de Ministros (PCM) a través de la Secretaría de Gestión Pública (PCM-SGP). La modernización —incluida la simplificación administrativa- es un proceso permanente caracterizado por una constante redefinición de sus objetivos y por su integración sistémica con las entidades públicas. Por eso, el proceso de modernización es considerado un sistema administrativo en el marco de las disposiciones de la Ley Orgánica del Poder Ejecutivo (LOPE).

Simplificación Administrativa y el Plan Nacional de Simplificación Administrativa, donde contabilizan 4815 mejoras traducidas en la simplificación de 3424 procedimientos administrativos. Aquí el detalle de las mejoras realizadas: 1588 reducciones de costos en procedimientos administrativos y servicios prestados en exclusividad; 1086 procedimientos administrativos y servicios prestados en exclusividad eliminados; 1387 requisitos eliminados en 729 procedimientos administrativos y servicios prestados en exclusividad; 534 procedimientos y servicios prestados en exclusividad con reducción en sus plazos de atención; y 220 procedimientos y servicios prestados en exclusividad con mejora en su calificación. 
La Ley del Procedimiento Administrativo General ${ }^{9}$ y el Reglamento de Organización y Funciones de la Presidencia del Consejo de Ministros (ROF-PCM) permiten identificar que el ente rector del sistema administrativo de modernización de la gestión pública — dentro del cual se incluye la materia de simplificación administrativa - es la PCM-SGP.

De acuerdo al artículo 36 del decreto supremo 063-2007-PCM (ROFPCM $)^{10}$, la Secretaria de Gestión Pública de la Presidencia del Consejo de Ministros (PCM-SGP) es el órgano de línea encargado de coordinar y dirigir el proceso de modernización de la gestión pública, y es competente en materia de funcionamiento y organización del Estado, de simplificación administrativa, de ética y transparencia en concordancia con las normas de modernización, de racionalización, de descentralización del Estado, de control interno y del funcionamiento del código de ética de la función pública.

Desde el 2007, como órgano rector, la PCM-SGP ha desarrollado los siguientes instrumentos del sistema:

- La política nacional de simplificación administrativa, aprobada mediante el decreto supremo 025-2010-PCM, que contiene los principios, objetivos y estrategias que deben cumplir todas las entidades públicas para brindar trámites y servicios administrativos valiosos y oportunos a la ciudadanía, sin dejar de dar relevancia a la optimización de procesos.

- El plan nacional de simplificación administrativa, aprobado por la resolución ministerial 228-2010-PCM y que busca la implementación de la política nacional de simplificación administrativa al desarrollar lo siguiente:

- Metodología de simplificación administrativa, aprobada por el decreto supremo 007-2011-PCM, el cual detalla los pasos, las herramientas y los métodos a utilizar por las entidades de la administración pública para desarrollar proyectos de reingeniería o simplificación administrativa en los procedimientos administrativos o servicios prestados en exclusividad.

9 Ley 27444, publicada en el diario oficial El Peruano el 11 de abril de 2001.

10 «Artículo $36^{\circ}$.- De la secretaría de gestión pública

La Secretaría de Gestión Pública es el órgano de línea que depende jerárquicamente de la Secretaría General, encargado de coordinar y dirigir el proceso de modernización de la gestión pública. La Secretaría de Gestión Pública es competente en materia de funcionamiento y organización del Estado, simplificación administrativa, ética y transparencia en concordancia con las normas de modernización, racionalización, descentralización del Estado, control interno, así como el código de ética de la función pública». 
- Metodología de determinación de costos de los procedimientos y servicios administrativos, aprobada por el decreto supremo 064-2010-PCM en cumplimiento del artículo 44, inciso 6, de la LPAG, el cual contiene la nueva metodología para la determinación de costos de los procedimientos administrativos y de servicios prestados en exclusividad.

- Instructivo de equipos de mejora continua. La PCM-SGP viene implementando Equipos de Mejora Continua (EMC) en entidades del Poder Ejecutivo que son grupos multidisciplinarios de servidores públicos, y en virtud a una formación técnica y administrativa ad hoc — proceso, metodologías, estadísticas, costos, análisis, reingeniería, innovación y solución de problemas, etcétera- se reúne periódicamente para definir, analizar y resolver los problemas que atañen a la entidad respecto de los procesos y los procedimientos administrativos.

- TUPA Modelo Urbano de Procedimientos Administrativos. TUPA simplificado para Municipalidades Provinciales y Distritales de ciudades capitales urbanas en seis procedimientos claves que son demandados con mayor frecuencia por los empresarios de la localidad: (i) licencia de funcionamiento para establecimientos de hasta $100 \mathrm{~m}^{2}$; (ii) licencia de habilitación urbana o de edificación; (iii) autorización de anuncio y paneles publicitarios; (iv) autorización para instalación de infraestructura necesaria para la prestación de servicios públicos de telecomunicaciones; (v) autorización de rotura de pistas y calzadas; y (vi) autorización para ocupación eventual de vía pública con fines comerciales.

Además, se está preparando un TUPA modelo rural, una guía de TUPA universitario, el sistema único de trámites y el sistema de trámite documentario.

Sin embargo, todos estos instrumentos solo serán eficaces si van unidos a un presupuesto por resultados de cada entidad pública que contenga indicadores de logros de simplificación administrativa y satisfacción ciudadana.

Asimismo, sería muy importante que las entidades públicas, por encima de esas metas, aprueben cartas de compromiso con la ciudadanía, las cuales estén publicadas en su portal al mismo tiempo que reportes periódicos sobre sus avances de cumplimiento. 


\section{LOS PROCEDIMIENTOS SANCIONADORES}

Un avance fundamental se ha producido en los procedimientos sancionadores que tienen principios propios aplicables a ellos, previstos en un capítulo de procedimientos especiales en la misma LPAG. Estos principios son particularmente garantistas por tratarse, justamente, de escenarios en los que se evalúa la comisión de infracciones y la aplicación de sanciones. Las diversas entidades públicas han ido adaptando la fisonomía de sus procedimientos sancionadores conforme a los principios y características de la LPAG, por lo que el Tribunal Constitucional ha desarrollado una importante jurisprudencia para orientar su debida aplicación. Asimismo, la doctrina nacional ha contribuido a precisar la interpretación de la LPAG y a analizar su vigencia ${ }^{11}$.

Recientemente, se ha modificado esta parte de la LPAG mediante el decreto legislativo $1029^{12}$. Los cambios o modificaciones introducidas por dicha norma son importantes para asegurar que la administración pública ejerza adecuadamente su función sancionadora, tal y como lo señala la doctora Liliana Tamayo: «[...] la modificación introducida por el DL 1029 al artículo 229 de la LPAG constituye un paso imprescindible para el correcto ejercicio de la actividad sancionadora de la Administración Pública en el Perú» ${ }^{13}$.

Dicha norma introduce modificaciones importantes, entre las que destacan las siguientes: (a) en los procedimientos establecidos en leyes especiales se deberán observar los principios de la potestad sancionadora prevista en el artículo 230 de la Ley de Procedimiento Administrativo General; y (b) se dispone que los procedimientos especiales no podrán imponer condiciones menos favorables a los administrados que las previstas en el capítulo sobre el Procedimiento Sancionador de la Ley de Procedimiento Administrativo General.

Sin embargo, queda pendiente perfeccionar algunos aspectos relevantes, tales como son: (i) la incorporación del principio de oportunidad entre los aplicables al procedimiento sancionador; y (ii) regular de manera expresa requisitos para la aplicación de medidas correctivas complementarias a las sanciones, que muchas veces son excesivas y sin oportunidad del afectado de defenderse ante su aplicación.

11 Dos textos muy útiles son (i) el de Juan Carlos Morón en su ya clásico y varias veces reeditado libro sobre la LPAG: MORÓN URBINA, Juan Carlos. "Comentarios a la Ley de Procedimiento Administrativo General». Gaceta Jurídica. Octava edición, título IV capítulo 2, 2009.; y (ii) los capítulos 8 y 9 sobre potestad sancionadora y procedimiento administrativo sancionador de MARAVí, Milagros (coordinadora). Sobre la Ley del Procedimiento Administrativo General. Libro homenaje a José Alberto Bustamante Belaunde. Lima: UPC, 2009.

12 Decreto legislativo que modifica la Ley del Procedimiento Administrativo General 27444 y la Ley del Silencio Administrativo 29060 y que fuese aprobado mediante el decreto legislativo 1029, publicado en el diario oficial El Peruano el martes 24 de junio de 2008.

13 TAMAYO, Liliana. «El procedimiento administrativo sancionador». En Milagros Maraví (coordinadora). Sobre La Ley del Procedimiento Administrativo General. Libro homenaje a José Alberto Bustamante Belaunde». Lima: UPC, 2009 p. 463.

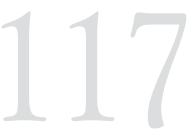

AVANCES A

LOS DIEZ AÑOS

DE VIGENCIA

DE LA LEY DEL

PROCEDIMIENTO

ADMINISTRATIVO

GENERAL

TEN YEARS

IN FORCE OF

THE GENERAL

ADMINISTRATIVE

PROCEDURE LAW:

PROGRESS 
Particularmente necesario nos parece el primer aspecto, ya que la función sancionadora es una más de las que cuentan las entidades públicas para gobernar —entre otras como la normativa, reguladora, supervisora de fiscalización, etcétera-y debe ser un instrumento para ese fin. Una característica de la función sancionadora que no está expresamente recogida en nuestra LPAG es su ejercicio facultativo. En efecto, la potestad sancionadora de la administración pública tiene por finalidad reprimir ciertas conductas en los administrados y desincentivar el incumplimiento del ordenamiento legal. Por eso, como señala Alejandro Nieto:

Frente a principio de legalidad, que implica el deber de perseguir y sancionarlas infracciones, el principio de oportunidad establece la posibilidad o permisividad de poner en marcha tales consecuencias jurídicas. $\mathrm{O}$ lo que es lo mismo: la Administración no está obligada por ley a castigar sino que simplemente se le autoriza a hacerlo. En los Derechos europeos el principio de oportunidad está absolutamente generalizado: en España por práctica indiscutida, lo mismo que en Francia (Mourgeon, 1967, 303 ss), y en Alemania por imperativo expreso del artículo 47.1 de la Ley Reguladora de las infracciones (OWING): La persecución de las infracciones depende de la discrecionalidad vinculada (pflicht Ermessen) de la Administración sancionadora, quien puede ordenar el archivo del expediente mientras el procedimiento sea de su competencia» ${ }^{14}$.

Como señala el mismo autor, la excepción «la no persecución» debe ser justificada, esto al iniciar o no expediente o decidir archivarlo en cualquier momento antes de la resolución. Por ejemplo, para no cambiar bruscamente de criterio y castigar infracciones que venían siendo toleradas o en situaciones en que medie culpabilidad leve y no estén en juego intereses públicos importantes.

\section{EL CONTROL DIFUSO DE LA ADMINISTRACIÓN}

En estos diez años se produjo un debate respecto de la facultad de las autoridades administrativas en lo relativo a no aplicar normas inconstitucionales o ilegales, respecto de lo cual el Tribunal Constitucional definió criterios relevantes en el precedente vinculante contenido en la sentencia 3741-2004-AA/TC del caso Salazar Yarlenque. El TC estableció que todo tribunal u órgano colegiado de la administración pública que imparte «justicia administrativa» con carácter nacional, adscrito al Poder Ejecutivo, tiene la facultad y el deber de preferir la Constitución y no aplicar una disposición infraconstitucional que la vulnera manifiestamente, bien por la forma, bien por el fondo. 
Esto de conformidad con los artículos 38, 51 y 138 de la Constitución. Para ello, se deben observar los siguientes presupuestos: (i) que dicho examen de constitucionalidad sea relevante para resolver la controversia planteada dentro de un proceso administrativo; y (ii) que la ley cuestionada no sea posible de ser interpretada de conformidad con la Constitución.

En la resolución aclaratoria precisa (puntos 7 y 8) que:

7. Excepcionalmente, el control difuso procede de oficio cuando se trate de la aplicación de una disposición que vaya en contra de la interpretación que de ella haya realizado el Tribunal Constitucional, de conformidad con el último párrafo del artículo VI del Título Preliminar del Código Procesal Constitucional; o cuando la aplicación de una disposición contradiga un precedente vinculante del Tribunal Constitucional establecido de acuerdo con el artículo VII del Título Preliminar del Código Procesal Constitucional.

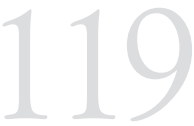

AVANCES A LOS DIEZ AÑOS DE VIGENCIA

DE LA LEY DEL PROCEDIMIENTO ADMINISTRATIVO GENERAL

TEN YEARS

IN FORCE OF

THE GENERAL ADMINISTRATIVE PROCEDURE LAW: PROGRESS

8. Que los tribunales administrativos y los órganos colegiados de la administración pública que imparten «justicia administrativa» con carácter nacional no pueden dejar de aplicar una ley o reglamento cuya constitucionalidad haya sido confirmada en procesos constitucionales, ni tampoco aplicar a las consecuencias de las relaciones y situaciones jurídicas existentes, en un caso concreto, los efectos jurídicos de una ley o reglamento que haya sido declarado inconstitucional en dichos procesos, de conformidad con el tercer párrafo del artículo VI del Título Preliminar del Código Procesal Constitucional.

En sugestivo artículo a este respecto titulado «Kelsen de cabeza: verdades y falacias sobre el control difuso de las normas por autoridades administrativas», publicado en la revista Themis ${ }^{15}$, Alfredo Bullard hace un análisis del dilema de enfrentar el principio de jerarquía con el de legalidad, y postula que tres elementos pueden ser considerandos para aplicar el control difuso en sede administrativa:

a) El carácter autárquico del órgano o Tribunal administrativo. Cuanto más independiente y autónomo sea, y cuanto más esté vinculada su actividad a la solución de conflictos intersubjetivos, más servirá el control difuso como una herramienta de garantía de los derechos ciudadanos y de límite al ejercicio del poder. b) La protección de derechos ciudadanos. Si el control difuso se usa para reforzar derechos ciudadanos (y no para debilitarlos) sus fines estarán plenamente alineados con los perseguidos por el principio de legalidad, es decir, de poner límites al gobernante. Un control difuso usa el principio de jerarquía para limitar 
al gobernante es consistente con el principio de legalidad. c) Carácter manifiestamente inconstitucional de la norma inaplicada. Cuando más evidente sea la violación, más evidente será que el emisor de la norma se excedió, por tanto los riesgos de excesos por parte de la autoridad que controla y protege la jerarquía de las normas serán menores ${ }^{16}$.

Es relevante haber definido estos criterios jurisprudenciales y doctrinarios para que las entidades públicas — justamente respetando la jerarquía de las normas - actúen conforme al principio de legalidad que manda:

Artículo IV.- Principios del procedimiento administrativo

1. El procedimiento administrativo se sustenta fundamentalmente en los siguientes principios, sin perjuicio de la vigencia de otros principios generales del Derecho Administrativo:

1.1 Principio de legalidad.- Las autoridades administrativas deben actuar con respeto a la Constitución, la ley y al derecho, dentro de las facultades que le estén atribuidas y de acuerdo con los fines para los que les fueron conferidas.

Es decir que la sujeción de los funcionarios públicos es no sólo a la ley sino primordialmente a la Constitución.

\section{EL SILENCIO ADMINISTRATIVO}

$\mathrm{Al}$ inicio de este escrito hemos citado las publicaciones de los congresos nacionales de Derecho administrativo que se han ocupado de reflexionar sobre el devenir del procedimiento administrativo general. Pues no es casual que en todas ellas se haya presentado ponencias vinculadas al silencio administrativo.

En el primer congreso analizamos la jurisprudencia del Tribunal Constitucional peruano respecto del silencio administrativo negativo y cómo se había definido jurisprudencialmente el concepto que corresponde al mismo, al entenderse como habilitador de impugnación si el administrado así lo decide y sin cómputo de plazos. Sin embargo, mucho más se ha discutido esta década sobre el silencio administrativo positivo. En el segundo congreso, Analía Calmell del Solar ${ }^{17}$ se refería a un tema debatido en doctrina y que representa un problema central: ¿qué ocurre si al aplicar el silencio administrativo positivo nos encontramos con un acto nulo? Citando a García Trevijano y Morell Ocaña indica que, mediante el silencio positivo, el particular no debería adquirir más — derechos e intereses- que los que la ley le permite o reconoce. La petición que da 
origen al procedimiento podrá entenderse estimada solo dentro de los límites que la ley establece. Por ejemplo, si el particular solicita una licencia para la construcción de una fábrica en una zona con zonificación residencial baja, no compatible con industria, no podrá entenderse -en ningún caso- que se ha obtenido una licencia. Sin embargo, advierte que en el caso peruano esta no es la opción, pues se presume la validez del acto presunto. Es decir, se sigue lo establecido por García de Enterría y T. Ramón Fernández, quienes señalan que si la administración considera que el acto administrativo generado adolece de vicio de nulidad deberá revisarlo de oficio declarando su nulidad.

El tercer congreso estuvo plagado de ponencias respecto del silencio administrativo positivo propiciadas por la aprobación de la ley 29060: Ley del Silencio Administrativo (LSA). Entre esas ponencias estaba la de Antonio Abruña y Víctor Baca, «El silencio administrativo en el Derecho peruano» ${ }^{18}$ que, vinculado con lo antes anotado por Analía Calmell, incide en la inseguridad que genera la técnica del silencio administrativo positivo por una diversidad de razones, entre las que destacan «a) la configuración amplísima de la invalidez, por la que los actos que resulten aprobados como consecuencia del silencio positivo son nulos de pleno derecho (artículo 10.3 LPAG)» «b) el régimen extremadamente sencillo de la revisión de oficio de los actos nulos de pleno derecho (artículo 188.2 y 202 LPAG)». Definitivamente, para la ciudadanía es muy riesgoso actuar sobre la base de actos administrativos obtenidos por silencio positivo, dado que no solo es difícil que sea reconocido por otras autoridades o incluso privados, sino porque sobre ellos pende una nulidad inminente pero incierta. Por eso, es importante difundir la práctica de entidades públicas de emitir actos confirmatorios de los producidos por silencio administrativo en beneficio de los solicitantes.

Entre otros autores, Abruña y Baca también cuestionaban el uso generalizado de la declaración jurada —que puede terminar siendo de exigencia obligatoria-. Esto mismo es señalado por Hugo Gómez y Ramón Huapaya ${ }^{19}$, quienes indican que se ha generado «una carga adicional para el administrado».

En «Lo bueno lo malo y lo feo de la Ley del Silencio Administrativo»20 también Gómez y Huapaya cuestionan que se haya sumado al plazo para resolver el de notificación, a efectos de que opere el silencio positivo. Esto abre un espacio para que $-\mathrm{y}$ se ha convertido en práctica

18 ABRUÑA, Antonio y Víctor BACA. «El silencio administrativo en el Derecho peruano». En El Derecho Administrativo y la modernización del Estado Peruano. Ponencias del Tercer Congreso Nacional de Derecho Administrativo. Lima: Grijley, 2008, p. 69.

19 GÓMEZ, Hugo y Ramón HUAPAYA. «Lo bueno, lo malo y lo feo de la Ley del Silencio Administrativo». En El Derecho administrativo y la modernización del Estado Peruano. Ponencias del Tercer Congreso Nacional de Derecho Administrativo. Lima: Grijley, 2008, p. 98.

20 Ob. cit., p. 97

AVANCES A

LOS DIEZ AÑOS

DE VIGENCIA

DE LA LEY DEL

PROCEDIMIENTO

ADMINISTRATIVO

GENERAL

TEN YEARS

IN FORCE OF

THE GENERAL

ADMINISTRATIVE

PROCEDURE LAW:

PROGRESS 
común - las entidades resuelvan fuera del plazo, cuando en realidad este espacio debiera estar reservado para notificar. Los ciudadanos, del otro lado del problema, no pueden probar que esto ha ocurrido así.

Finalmente, en el cuarto y último congreso, Juan Carlos Cassagne en «Las vicisitudes del silencio administrativo» ${ }^{21}$, con la sabiduría que lo caracteriza y sin el apasionamiento que generaba la reciente aprobación de la LSA, indicaba que la técnica del Silencio Administrativo Positivo (SAP) no debía generalizarse por los inconvenientes para la seguridad jurídica que plantea desde la obtención del certificado que acredita el acto presunto hasta la revisión de oficio de un acto administrativo sin los elementos que aseguren la vigencia del principio de legalidad — verbigracia, «falta de motivación en el objeto, causa y finalidad del acto»Asimismo, indicaba que no debería extenderse el SAP a los procedimientos recursivos ${ }^{22}$ "que se circunscriban a los supuestos excepcionales que se justifican frente a la inactividad material de la Administración, en los que hay un interés privado que puede satisfacerse a través de un acto administrativo presunto, sin afectar el orden público administrativo».

El mismo actor concluye que:

La pretensión de superar el problema de la inactividad material a través de la figura del acto presunto o silencio administrativo positivo resulta ilusoria y que los problemas que trae consigo su generalización implica serios riesgos a la seguridad jurídica. Lo que hay que hacer es mejorar los medios humanos y materiales con que cuenta la Administración. Sin funcionarios que acrediten idoneidad técnica y moral, con una adecuada vocación de servicio, la introducción del SAP será un estímulo para no hacer y eludir las responsabilidades consiguientes, dejando en una suerte de limbo jurídico la obligación de resolver que constituye la esencia de una buena y eficaz administración, la cual, como cualquier otra obligación, es siempre correlativa ${ }^{23}$.

Esto está vinculado con nuestras reflexiones en torno a la simplificación administrativa en este mismo artículo.

El devenir del silencio administrativo no ha sido auspicioso, entonces, para esta institución misma, sino fuese por el efecto colateral de promover procesos de simplificación administrativa en todas las entidades públicas. 


\section{CONCLUSIÓN}

El recuento y análisis anterior, además de los avances o problemas de la LPAG, nos revela la presencia creciente de doctrina y jurisprudencia de Derecho administrativo — cada vez más especializada — y es que esta década también ha significado el crecimiento y la consolidación de la especialidad de Derecho administrativo en el país, cosa que debe ser celebrada por todos los que trabajamos en ella, pero, más aún, por el impacto positivo que esto trae en el desempeño de las entidades públicas en resguardo del interés público y para beneficio de la ciudadanía en general.

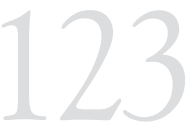

AVANCES A

LOS DIEZ AÑOS

DE VIGENCIA

DE LA LEY DEL

PROCEDIMIENTO

ADMINISTRATIVO

GENERAL

TEN YEARS

Recibido: 14-07-2011

IN FORCE OF

Aprobado: 08-08-2011

THE GENERAL

ADMINISTRATIVE

PROCEDURE LAW:

PROGRESS 